\title{
Resource Allocation using Deep Learning in Mobile Small Cell Networks
}

This paper was downloaded from TechRxiv (https://www.techrxiv.org).

\section{LICENSE}

CC BY 4.0

SUBMISSION DATE / POSTED DATE

02-02-2021 / 04-02-2021

\section{CITATION}

Zafar, Saniya; Jangsher, sobia; Al-Dweik, Arafat (2021): Resource Allocation using Deep Learning in Mobile Small Cell Networks. TechRxiv. Preprint. https://doi.org/10.36227/techrxiv.13685947.v1

DOI

10.36227/techrxiv.13685947.v1 


\title{
Resource Allocation using Deep Learning in Mobile Small Cell Networks
}

\author{
Saniya Zafar, Sobia Jangsher Member, IEEE, and Arafat Al-Dweik, Senior Member, IEEE
}

\begin{abstract}
The deployment of mobile-Small cells (mScs) is widely adopted to intensify the quality-of-service $(\mathrm{QoS})$ in high mobility vehicles. However, the rapidly varying interference patterns among densely deployed $\mathrm{mScs}$ make the resource allocation (RA) highly challenging. In such scenarios, RA problem needs to be solved nearly in real-time, which can be considered as drawback for most existing RA algorithms. To overcome this constraint and solve the RA problem efficiently, we use deep learning (DL) in this work due to its ability to leverage the historical data in RA problem and to deal with computationally expensive tasks offline. More specifically, this paper considers the RA problem in vehicular environment comprising of city buses, where DL is explored for optimization of network performance. Simulation results reveal that $\mathrm{RA}$ in a network using Long ShortTerm Memory (LSTM) algorithm outperforms other machine learning (ML) and DL-based RA mechanisms. Moreover, RA using LSTM provides less accurate results as compared to existing Time Interval Dependent Interference Graph (TIDIG)-based, and Threshold Percentage Dependent Interference Graph (TPDIG)based RA but shows improved results when compared to RA using Global Positioning System Dependent Interference Graph (GPSDIG). However, the proposed scheme is computationally less expensive in comparison with TIDIG and TPDIG-based algorithms.
\end{abstract}

Index Terms-Deep Learning (DL), Long Short-Term Memory (LSTM), mobile-Small cells (mScs), Quality-of-Service (QoS), Resource Allocation (RA), Resource Blocks (RBs).

\section{INTRODUCTION}

Mobile communication systems were introduced a few decades ago, where the main goal was to provide voice-only services to the users to a limited extent. For an instant, first generation (1G) networks in 1990 were supporting about 11 million users worldwide [1]. Current generations of wireless standards such as the fourth and fifth generations $(4 \mathrm{G})$ and (5G) respectively, have to support about 6.1 billion users in 2020 [2], [3], i.e., about 550 fold increase. Nevertheless, the increased number of users is not the main challenge if such networks have to support voice-only services because the bandwidth, power as well as quality of service (QoS) requirements are manageable for voice centric applications. The main challenge is that current networks should support data services with high data rates for large number of users, which created

S. Zafar is with Wireless and Signal Processing Lab, Institute of Space Technology Islamabad, Pakistan, (E-mail: saniya.zafar07@ist.edu.pk).

S. Jangsher is with Wireless and Signal Processing Lab, Institute of Space Technology Islamabad, Pakistan, (E-mail: sobia.jangsher@ist.edu.pk).

A Al-Dweik is with the Center for Cyber Physical Systems, Khalifa University, AbuDhabi, UAE, (Email: arafat.dweik@ku.ac.ae). Also, he is with the Department of Electrical and Computer Engineering, Western University,London, ON, Canada, (E-mail: dweik@ fulbrightmail.org). a severe shortage of transmission resources. Moreover, the demand for data services is continuously increasing as shown by the latest statistics of wireless networks, which revealed that data traffic for mobile users grew $68 \%$ in 2019, reaching 38 Exabytes (EB) per month as compared to $27 \mathrm{~EB}$ per month in 2018 [2], [3]. Furthermore, Ericsson predicts that the mobile data traffic will show an upsurge of $27 \%$ annually till 2025 [2]. Consequently, it will be insufficient to focus on a particular network aspect to improve, instead, future wireless generations should optimise all system parameters to be able to meet the escalating demands for data and high QoS requirements. Network densification and efficient resource allocation are among the prominent techniques that can be used to increase the network efficiency [4].

Network densification (NeDe) is considered as a pivotal solution for increasing the capacity of wireless networks, and it is an integral part of the 5G architecture [5]. The main concept of NeDe is to deploy low powered small cells in large amounts in order to complement the macrocell functionality in areas with poor signal quality, such as indoor environments [6]. The term small-cell covers a variety of cell types such as minicells, micro-cells, pico-cells, and femto-cells. When a smallcell is incorporated in a vehicle, it is denoted as mobile-Small cell $(\mathrm{mSc})$. One of the main advantages of deploying $\mathrm{mScs}$ is that they mitigate the significant attenuation of wireless signals when they propagate through the thick metal body of the vehicle. Moreover, the distance between the $\mathrm{mSc}$ access point $(\mathrm{mScAP})$ and its associated mobile users is decreased, which in turn enhances the QoS inside the vehicle [6]-[8].

Resource allocation (RA) aims at distributing the transmission resources among different network users, and if the RA strategy is performed optimally, it may impact the performance of a network significantly in terms of capacity and satisfaction of users QoS demands [9]-[12]. The synergy of NeDe and RA would be highly beneficial, however the RA process has to be optimized specifically for small-cell networks. Therefore, RA for small-cell networks have received significant interest by the research community as reported in [4], [13]-[15], and the references listed therein. Moreover, because cellular users connected from mobile vehicles are among the primary network users, resource allocation for $\mathrm{mSc}$ networks has been considered as well [6], [16], [17].

Unlike macrocell and static small-cell networks, RA for $\mathrm{mScs}$ is more challenging because of the severe time-varying channel conditions [18], the high handover frequency between $\mathrm{mScs}$ and macro-cells, and between the users and $\mathrm{mScs}$ [16]. The cell mobility in dense cellular network also introduces irregular time-varying interference patterns between $\mathrm{mScs}$ in 
close proximity of each other. Consequently, satisfying QoS requirements for users connected to $\mathrm{mScs}$ typically requires allocating more transmission resources as compared to static small-cell networks, and the RA has to be performed more frequently. Therefore, adopting efficient RA strategies is indispensable to perform RA under such stringent constraints.

\section{A. Related work}

Although the problem of RA has been thoroughly investigated in the literature [4], [9]-[15], [19], little work focused on RA for mScs. Typically, RA for $\mathrm{mScs}$ is performed using mathematical programming, which involves either maximizing the sum rate or minimizing the interference between $\mathrm{mScs}$ in the network [6], [8], [20]. For example, Xiao et al. [19] proposed a non-cooperative game theory mechanism that considers the vehicular mobility characteristics for RA in vehicular heterogeneous networks. The work in [6]-[8] considers RA in vehicles travelling at a uniform speed along a fixed path, and [16], [17], [20] consider the case of variable speed following a predefined path. However, these works can not handle the diverse requirements of mobile users for highly dense $\mathrm{mScs}$. Although some of the aforementioned RA mechanisms may provide noteworthy results for $\mathrm{mSc}$ networks, it is nearly infeasible for most of them to handle the exact required services for all users in real-time. Therefore, a more flexible framework is required for RA in $\mathrm{mSc}$ networks.

Recently, Deep Learning (DL) has gained immense momentum where it has been used in the context of RA with the goal of providing RA solutions in real-time [21], [22]. The authors of [23] proposed a deep Q-learning mechanism for joint RA of networking, computing, and caching in software defined satellite terrestrial networks. Wu et al. [24] described a mechanism for empowering mobile devices that are capable of adapting to dynamic RA using commercial-off-the-shelf based DL systems. In [25], the authors addressed the RA problem relevant to network slicing at network provider's end. The proposed model enables network operators to slice various resources among diverse user cases efficiently. Zhao et al. [26] described a distributed deep reinforcement learning-based platform for optimal association of user equipment to base stations and allocation of radio resources to user terminals in heterogeneous networks. Power allocation in wireless networks (considering time division multiple access (TDMA)) using deep reinforcement learning is suggested in [27]. The authors of [28] formulated the maximization problem of weighted throughput while allocating the power as resource in wireless network using deep neural network architecture. The work in [29] focuses on the development of vehicular edge computing network where the vehicles serve as the edge computing servers for user devices. Reinforcement learning is utilized by the authors to obtain the optimal RA to vehicular edge computing systems. Double deep Q-learning network-based allocation of computation as well as communication resources is proposed in [30] for edge computing networks comprising of a number of edge servers and mobile devices to achieve low latency and balancing the load. The authors of [31] considered the context centric Internet-of-Things (IoT) environment for green RA using deep reinforcement learning under various transmission rates to enhance the quality of experience $(\mathrm{QoE})$ for users.

Similarly, from the perspective of $\mathrm{mSc}$ network, various recent works [22], [32] has demonstrated that significant performance gain can be achieved by adopting DL. Liang et al. [22] provides a comprehensive review unleashing the potentials of DL and deep reinforcement learning-based RA over the traditional RA mechanisms in wireless communications. The authors also highlighted the potential of DL for RA problems in generalized vehicle-to-vehicle (V2V) communication networks. In [32], autonomous allocation of resource blocks (RBs) and transmission power using multi agent deep reinforcement learning is presented for train-totrain communication, and communication between vehicles travelling with deterministic mobility. The authors of [16], [17] examined the allocation of resources in a mSc network and mobile IoT (mIoT-mSC) networks, respectively. More specifically, [16] targeted optimizing the RB allocation by minimizing the interference between $\mathrm{mScs}$, while [17] considered maximizing the data rates in mIoT-mSC networks. In these works, DL is used as an initial step for interference determination between the $\mathrm{mScs}$, and later, the resources are allocated using mathematical approaches that require accurate and real-time $\mathrm{mSc}$ information. The main drawback of such techniques is that acquiring real-time information becomes infeasible for large number of $\mathrm{mSCs}$, and the RA using DL in probabilistic mobility-based scenarios is not exploited properly.

\section{B. Motivation and main contributions}

With the emergence of several new applications, it is becoming harder to address the diverse requirements of mobile users using conventional methods, which are computationally expensive and are difficult to be applied in real-time. Therefore, we consider DL-based strategy to deal with the shortcomings of the traditional approaches. DL has shown an upsurge in various research domains because of its outstanding performance in several aspects such as speech recognition, face recognition, object detection, etc. DL has become able to deal with problems that are difficult to solve using conventional methods due to the availability of increased computational power and enhancement in training techniques,. As DL is a powerful data driven mechanism, it can be applied for RA by solving complex optimization problems [22].

Therefore, an efficient DL-based RA is proposed in this work to satisfy the QoS requirements in a city bus transit system. Overall, the contributions of the paper are:

- Examine the deployment of $\mathrm{mScs}$ in nearly realistic scenario of city buses associated to different bus routes.

- Formulate a data set comprising of spectrum data [33] using the vehicular network tool box and utilize the graph theory [34] to optimally allocate the RBs to all the mScs present in the data set at each time instant to achieve the ground truth labels.

- Propose a DL-based Long Short-Term Memory (LSTM) [35] algorithm for RA in mSc networks such that the demands of each cellular user is fulfilled. 


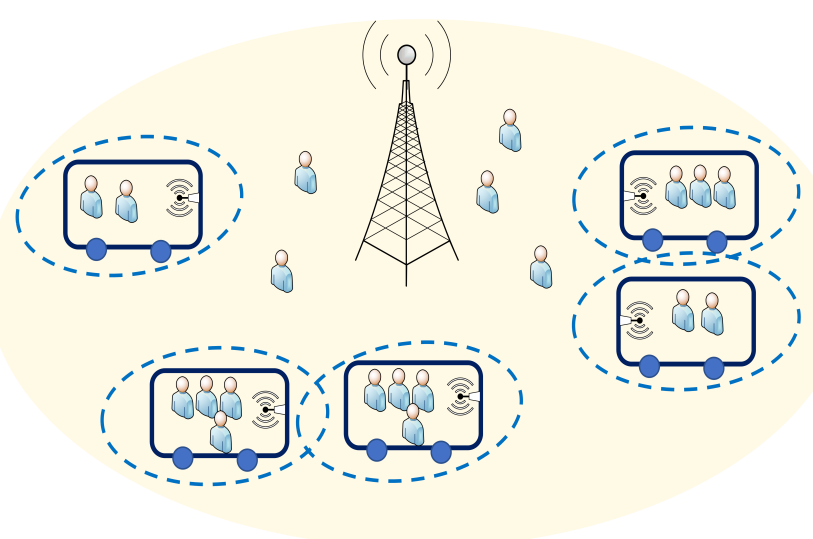

Fig. 1. System model of macrocell-mSc network.

- Simulation results of proposed LSTM-based RA method is compared with DL-based and conventional RA mechanisms.

\section{Paper Structure}

The rest of the paper is arranged as: Section II explains the system model of mSc network and Section III specifies the optimization problem. Section IV proposes the DL-based resource allocation method and Section V demonstrates the respective simulation results. Section VI provides the conclusion of paper.

\section{SYSTEM MODEL}

The mobile dense cellular network comprises of a macrocell and a number of mScs. Fig. 1 shows an example of macrocell-mSc network in order to get the meaningful insights while allocating the resources using DL. The network model considers the incorporation of $M \mathrm{mScs}$ in $B$ city buses travelling along $S$ routes. $\mathcal{S}=\{1,2, \ldots, S\}$ and $\mathcal{B}=\{1,2, \ldots, B\}$ represent the set of routes and set of city buses, respectively. The total number of buses moving on a single route $r$ is denoted as $B_{r}$. The letter $j$ is used to denote an $\mathrm{mSc}$ in a network, where $j \in\{1,2, \ldots, M\}$. Each city bus is equipped with a single $\mathrm{mSc}$ and the ID assigned to each $\mathrm{mSc}$ is unique. The $j$ th $\mathrm{mSC}$ serves $U_{j}$ users, forming total of $U$ users served by all $\mathrm{mScs}, U=\sum_{j=1}^{M} U_{j}$. All $\mathrm{mScs}$ are associated to macrocell, and macrocell is linked to the core network via macro-cell backhaul link.

The $\mathrm{mScs}$ affiliated to the macrocell-mSc network are using orthogonal frequency division multiple access (OFDMA) technology [36]. OFDMA forms time-frequency slots by dividing the time and frequency [36]. Each time-frequency slot is considered as an RB, which is the smallest resource element that can be allocated. The network is assumed to have a total of $K \mathrm{RBs}$, where $\mathcal{K}=\{1,2, \ldots, K\}$ represents the set of RBs. Moreover, transmit power associated with each RB is assumed to be equal for all RBs. The minimum demand of each $\mathrm{mSc}$ is denoted as $D_{j}$. Table. I provides all the parameters and their description associated with the system model. The transmission time frame $T$ is detached into $n$ time slots and each of the time slot is of equal length $\Delta t$. The time slots
TABLE I

SYSTEM MODEL PARAMETERS

\begin{tabular}{|l|l|}
\hline Parameter & Description \\
\hline$M$ & Total mobile Small cells in a network \\
\hline $\mathcal{B}$ & Set of city buses \\
\hline $\mathcal{S}$ & Set of city bus routes \\
\hline$B_{r}$ & Total city buses associated to route $r$ \\
\hline$U$ & Total mobile users in a network \\
\hline$U_{j}$ & Total users connected to mSc $j$ \\
\hline$K$ & Total resource blocks \\
\hline$D_{j}$ & Minimum demand of a mSc $j$ \\
\hline$n$ & Total time slots in time period \\
\hline$\Delta t$ & Length of a time slot \\
\hline $\mathcal{I}_{j}$ & Interference set associated to a mSc $j$ \\
\hline
\end{tabular}

are discrete in nature, and each slot is represented by $t_{n}$ for a whole time period. $\Delta t$ can be determined as: $\Delta t=t_{n}-t_{n-1}$. Fig. 2 shows the time slots formation in a given time period, the channel is considered as quasi-static.

For successful transmission in dense macrocell-mSc dense network, the interference level should not exceed a certain threshold. Therefore, interference between $\mathrm{mScs}$ should be managed using a particular interference control protocol [37]. According to [16], the RB interference constraint not only improves the QoS provided to a $\mathrm{mSc}$ user, but also enhances the overall performance of $\mathrm{mScs}$ network.

The number of $\mathrm{mScs}$ present within the interference range of a $\mathrm{mSc} j$ are the interfering $\mathrm{mScs}$, and form an interference set $\mathcal{I}_{j}$ for $\mathrm{mSc} j$. The signal to interference-plus-noise ratio (SINR) of mobile user $u$ affiliated with $\mathrm{mSc} j$ within time slot $t$ is:

$$
\operatorname{SINR}_{u, j}^{k}(t)=\frac{z_{u, j}^{k}(t)\left|h_{u, j}^{k}(t)\right|^{2} p_{u, j}^{k}(t) d_{u, j}^{-\beta}(t)}{N_{0}+\sum_{\substack{m=1 \\ m \neq j}}^{M} z_{u, m}^{k}(t) p_{u, m}^{k}(t)\left|h_{u, m}^{k}(t)\right|^{2} d_{u, m}^{-\beta}(t)}
$$

where $z_{u, j}^{k}(t)$ is the RA indicator which manages the allocation of resources to $\mathrm{mSc} j$ indicating either $\mathrm{RB} k$ is allocated to serve user $u$ of $\mathrm{mSc} j$ or not. This can be described by:

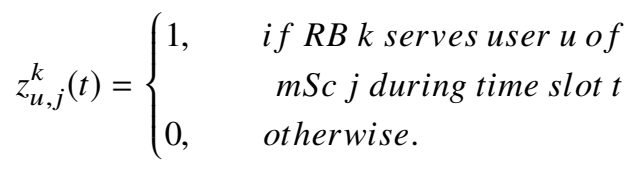

where $p_{u, j}^{k}(t)$ represent the transmission power of $\mathrm{mSc} j$ to user $u$ that is considered equal for all the $\mathrm{mScs}, h_{u, j}^{k}(t)$, is the channel gain that follows the Rayleigh distribution that evolves with time $t$, and $N_{0}$ is the power spectral density of additive white Gaussian noise (AWGN). The term

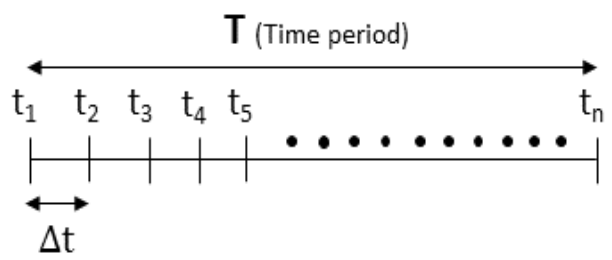

Fig. 2. Time slot formation in time period. 
$\sum_{\substack{m=1 \\ m \neq j}}^{M} z_{u, m}^{k}(t) p_{u, m}^{k}(t)\left|h_{u, m}^{k}(t)\right|^{2} d_{u, m}^{-\beta}(t)$ is the interference from all other mScs to user $u$ of $\mathrm{mSc} j$ over a RB $k$ in time slot $t$.

Therefore, the data rate of $\mathrm{mSc} j$ transmitting to user $u$ on RB $k$ during a time slot $t$ can be expressed as:

$$
R_{u, j}^{k}(t)=W \log _{2}\left(1+\operatorname{SINR}_{u, j}^{k}(t)\right)
$$

where $W$ represents the allocated bandwidth. Furthermore, for all RBs allocated to $\mathrm{mSc} j$ transmitting to user $u$ in a given time slot $t$, the downlink data rate of an access link can be calculated as:

$$
R_{u, j}(t)=\sum_{k=1}^{K} R_{u, j}^{k}(t)
$$

the data rate achieved for the downlink transmission of $\mathrm{mSc} j$ to all associated users in time slot $t$ is:

$$
R_{j}(t)=\sum_{u=1}^{U_{j}} R_{u, j}(t)
$$

and the overall data rate of all the $\mathrm{mScs}$ in a time slot $t$ is:

$$
R(t)=\sum_{j=1}^{M} R_{j}(t)
$$

Finally, the data rate of an access link of a downlink $\mathrm{mSc}$ network for all time periods is given by:

$$
R=\sum_{t=1}^{T} R_{j}(t)
$$

\section{PROBLEM FORMULATION FOR RESOURCE ALLOCATION}

In this section, the mathematical formulation of allocating $K \mathrm{RBs}$ to $M \mathrm{mScs}$ is presented. The objective is to allocate the resources in a dense $\mathrm{mSc}$ network in order to minimize the utilization of RBs such that the minimum demand of all the mobile users are fulfilled. The objective function is:

$$
\begin{array}{clc}
\underset{Z}{\operatorname{minimize}} & \sum_{t=1}^{T}\left(\sum_{k=1}^{K} \bigcup_{j=1}^{M} \bigcup_{u=1}^{U} z_{u, j}^{k}(t)\right) \\
\text { subject to } & \sum_{j \in \mathcal{I}_{j(t)}} z_{u, j}^{k}(t) \leq 1 \quad \forall j, k, t, \\
& D_{j}(t) \leq \sum_{k=1}^{K} z_{u, j}^{k}(t) \quad \forall j, t, \\
& p_{u, j}^{k}(t)>0 & \forall u, j, k, t ., \\
z_{u, j}^{k}(t) \in\{0,1\} & \forall u, j, k, t .
\end{array}
$$

where $z_{u, j}^{k}(t)$ represents the $\mathrm{RB}$ allocation variable as discussed in (2). The constraints related to the objective function are provided in $(8 \mathrm{~b})-(8 \mathrm{e})$. The constraint mentioned in $(8 \mathrm{~b})$ ensures that the RB assigned to a specific mSc $j$ cannot be allocated to any other $\mathrm{mSc}$ present in the interference range of that $\mathrm{mSc}$ in specific time slot $t$. In $(8 \mathrm{c})$, the constraint associated to the satisfaction of $\mathrm{mSc}$ demands is presented. This constraint assures that the minimum demand of each $\mathrm{mSc}$ will be satisfied. (8d) is the non-negative constraint that forces

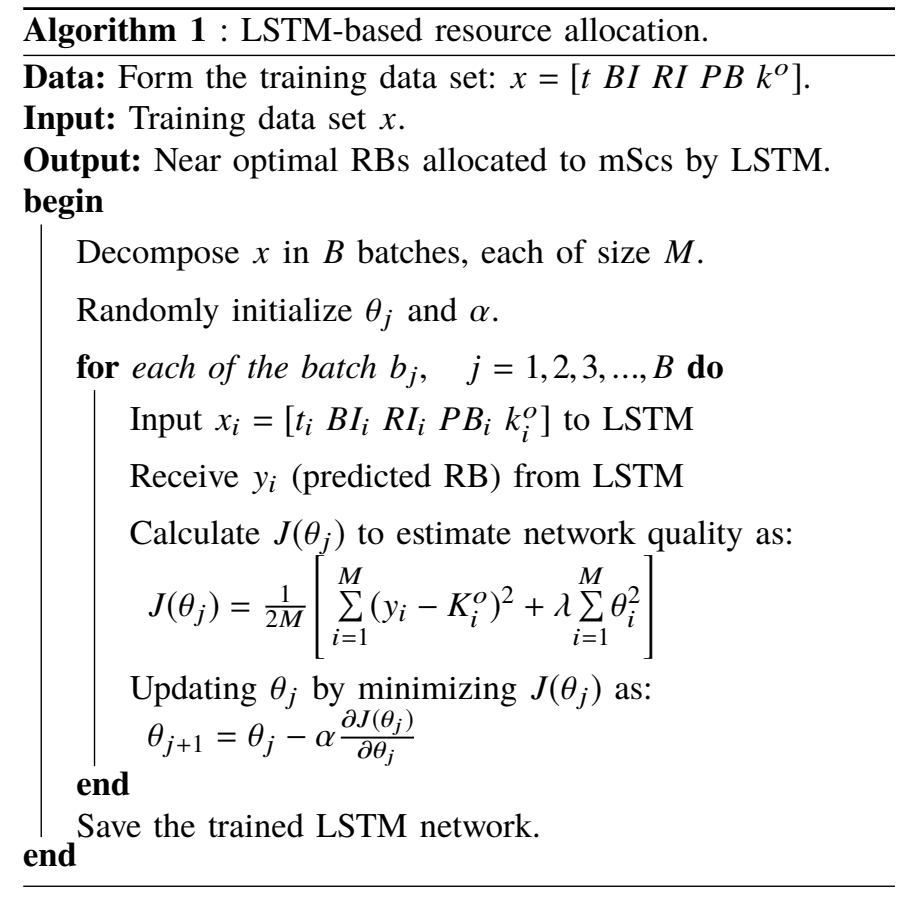

the power of the $\mathrm{mSc}$ to be positive. The last constraint (8e), is the binary variable constraint.

It can be noticed from the formulated problem that the considered minimization problem is NP-hard, and the interference part of the formulated problem makes it non-convex. Consequently, the RA is a challenging task because finding the optimal solution for a non-convex problem is difficult. Because the problem cannot be solved mathematically, a DL-based approach is proposed. A DL-based mechanism is considered in this paper due to its universal capability of approximating the relationship among the given input parameters and the respective optimal solution.

\section{DEEP LEARNING BASED RESOURCE ALLOCATION ALGORITHM}

This section presents the DL-based LSTM algorithm for RA where near-optimal solution can be obtained for the formulated optimization problem. In mobile dense cellular network, we consider a centralized processing mechanism in which the central hub collects the necessary information about all $\mathrm{mScs}$ including the communication overheads between them. All the processing required for the resource allocation is performed at the central hub and the available RBs are allocated to the $\mathrm{mScs}$ based on their demand for traffic.

We consider the supervised DL mechanism called LSTM for our formulated problem. We leverage DL-based LSTM algorithm in order to assign the resources in a mSc network by learning the relationship between inputs and outputs of a problem while treating the formulated problem as a black box. In the proposed methodology, conventional optimization mechanism is taken into consideration which will act as a supervisor in the algorithm. The output of the conventional approach will be presented as ground truth labels for LSTM, which has the ability of universal approximation as it maps the input parameters to the respective outputs [35]. Fig. 3 

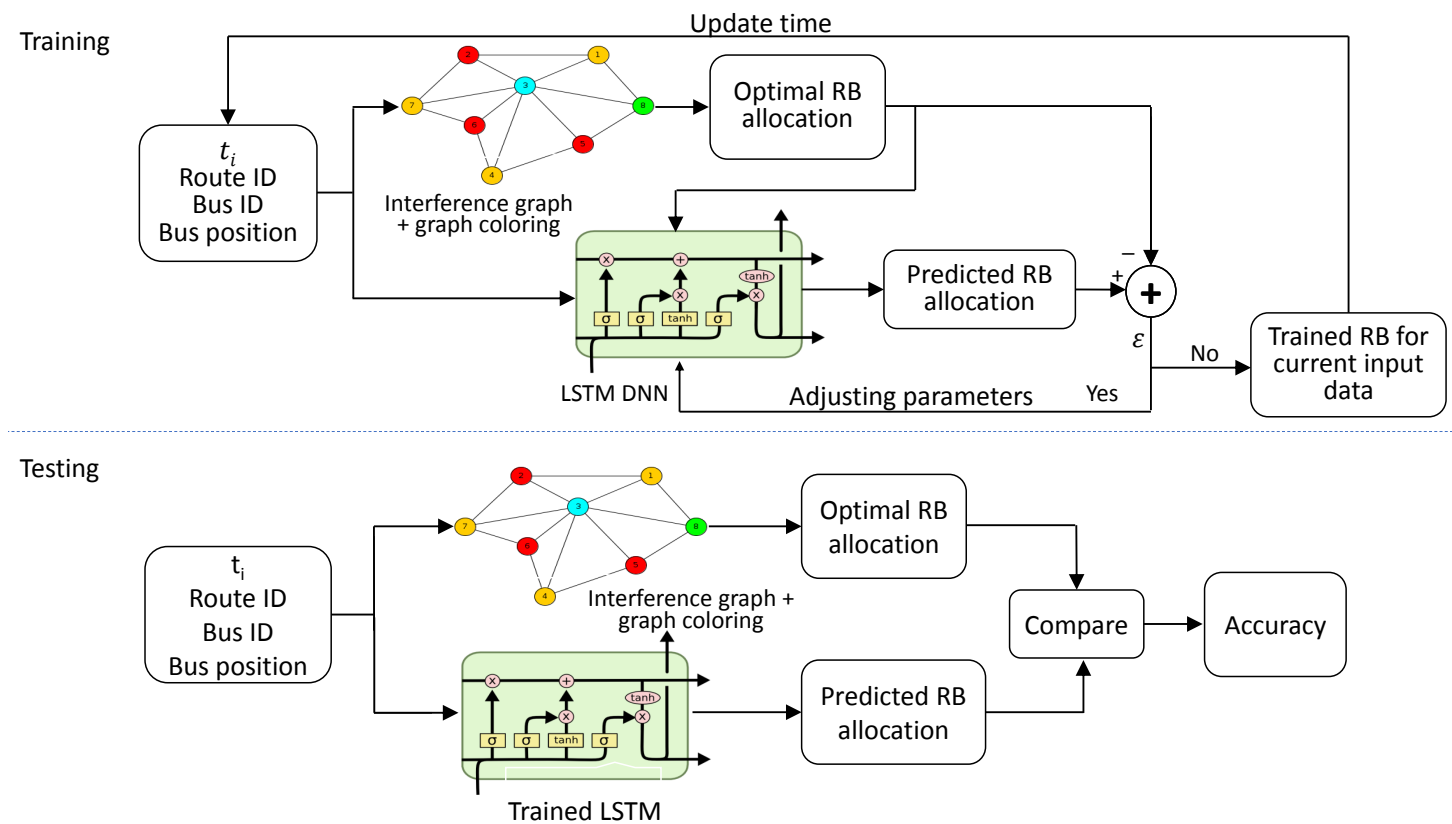

Fig. 3. LSTM-based resource allocation in mSc network.

demonstrates the training and testing phases of the proposed resource allocation mechanism. Both phases are discussed in Subsection IV-A and Subsection IV-B.

\section{A. Training phase}

Algorithm 1 presents the training phase of resource allocation using DL-based LSTM mechanism. For training the supervised network, labeled samples $k^{o}$ are required for the corresponding historic data $h=\left[\begin{array}{llll}t & B I & R & P B\end{array}\right]$. The variable $t$ represents the time vector with the corresponding bus ID vector $B I$, route ID vector $R I$, and position vector of buses $P B$. The vector $k^{o}$ is the vector of labels associated to the historic data $h$ and is formed by running the optimization algorithm which uses the historic data $h$ as presented in Fig.3. For creating the labels $k^{o}$ corresponding to historic data $h$, optimization algorithm involves the generation of time dependent interference graph and applying graph coloring algorithm to the generated graph [17]. Now, we have training data set $x$ comprising of the mentioned historic input data vectors $h$ along with their ground truths $k^{o}$, where $x=\left[\begin{array}{lllll}t B I & R I & P B & k^{o}\end{array}\right]$. Data set $x$ is taken as an input to train the LSTM network for producing the corresponding output vector $y$, where $y$ is the predicted vector of RBs for the respective mScs.

Once the data set is formed and input is defined, the algorithm begins as mentioned in algorithm 1 . As, the training phase depends on the size of mini-batches associated to Gradient Descent (GD) algorithm. Therefore, the training data set $x$ is first decomposed into total of $B$ batches and size of each batch $b_{j}$ is $M$ as described in next line of algorithm 1 . The later step mentioned in the algorithm is to randomly initialize the weights $\theta_{j}$ as well as the learning rate $\alpha$. As the input data set is divided into number of batches so, next line of algorithm applies for loop on each batch $b_{j}$ where $j=1,2,3, \ldots, B$. In later line i.e. inside the loop, for all $i$ ( $i$ represents the specific

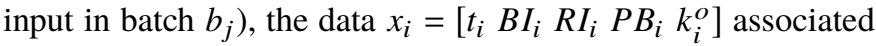
to $b_{j}$ is taken as an input to LSTM and respective output $y_{i}$ i.e. the predicted RB associated to $i^{t h}$ input of batch $j$ is received as an output from LSTM that is presented in next line of algorithm 1. Afterwards, the loss function for the batch $b_{j}$ by calculating the discrepancy present between the results achieved using LSTM and the ground truth labels is calculated using the eq. 9 in order to determine the quality of trained network as described in next line.

$$
J(\theta)=\frac{1}{2 M}\left[\sum_{i=1}^{M}\left(y_{i}-K_{i}^{o}\right)^{2}+\lambda \sum_{i=1}^{M} \theta_{i}^{2}\right] .
$$

Here, $y_{i}$ is the $i^{t h}$ output of LSTM and $K_{i}^{o}$ represents the ground truth labels that is optimal output received by the the traditional method. The summation is done over $i=1,2, \ldots, M$. Afterward, next line shows that LSTM updates the weights $\theta_{j}$ for each of the batch $b_{j}$ by lessen the achieved loss function in the preceding line. The weights are updated with the help of GD mechanism using $\theta_{j+1}=\theta_{j}-\alpha \frac{\partial J\left(\theta_{j}\right)}{\partial \theta_{j}}$. where: $\alpha$ represents the learning rate of the LSTM. Once the network is trained for all the batched of training data, the trained network is saved as given at the end of algorithm 1. With the help of DL-based LSTM algorithm, both the computational and implementation complexity is reduced for RA in mSc network. In conventional RA mechanism, the computational complexity is $\mathrm{O}\left(U^{2} L+\right.$ $\sigma U)$ in which $U^{2} L$ represents the complexity of RA to TIDIG using greedy approach and $\sigma U$ is the computational complexity of the QoS improvement algorithm [17]. $\sigma$ depicts the total iterations required to achieve $D_{j}$ of mSc. Contrary to that, the computational complexity of LSTM network is $\mathrm{O}\left(M^{2} N\right)$, where: $M$ represents the total volume of training data and $N$ represents the dimension associated to the training 
- $R B s$ requirement of $m S c s$

Velocity of city buses
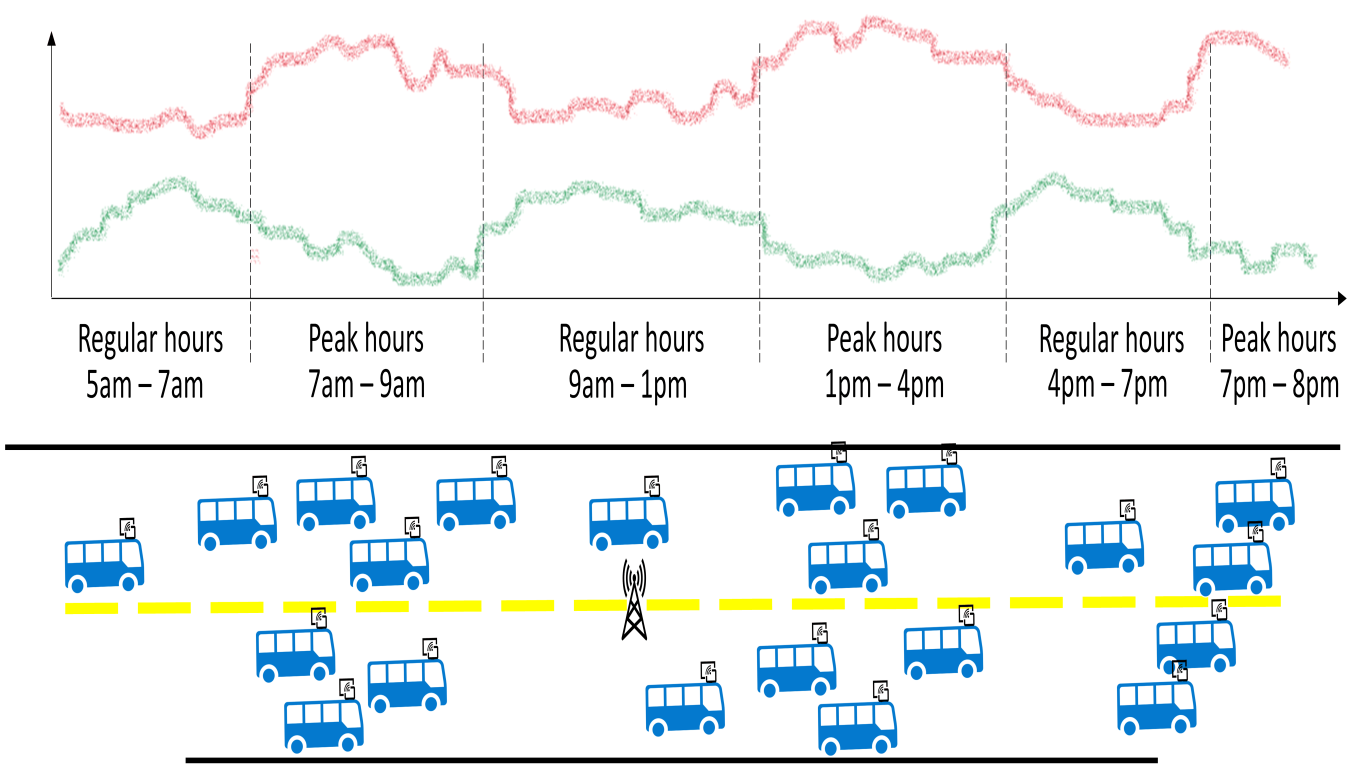

Fig. 4. City bus transit system peak-hour and regular-hour scheduling.

data set.

\section{B. Testing phase}

For testing phase, the sample labels are created using the same conventional approach that is used in the training phase as shown in Fig. 3. Afterwards, the input vectors are given to the trained LSTM network and the corresponding outputs are collected. Then, the comparison of the inferred solutions and the respective ground truths are compared to evaluate the trained LSTM.

\section{PERFORMANCE EVALUATION}

We considered software based generation of historic data and implement the proposed resource allocation algorithm in the same software. Historic data plays a fundamental role in allocation of resources to $\mathrm{mScs}$ using deep leaning-based LSTM algorithm. Toolbox named vehicular network is used for the formation of historic data [16], [17]. The mobility characteristics of city buses involving the speed and path (route) information along with a number of other factors covering road conditions, weather, traffic peak hours, etc. impacts the city bus system. The speeds and routes of city buses are paid special attention while designing the city bus system in a software because the city buses travel along different routes and any two buses may or may not share the same road segments resulting in dynamic interference patterns. Fig. 4 discusses the peak hour and regular hour scheduling of the modeled city bus transit system in software and its effect on the velocity concerned with the city buses as well as its impact on the utilization of resources to $\mathrm{mScs}$ placed in city buses. It can be seen in figure that during the regular hours of traffic, city buses travel with higher speed whereas the RBs allocated to the $\mathrm{mScs}$ equipped in city buses are lower in number because the interference between the $\mathrm{mScs}$ is less. Contrary to this, the speed of city buses is reduced in the peak hours of traffic and the requirement of resources increases as more $\mathrm{mScs}$ comes in the interference range of each other. We considered most of the factors while designing the city bus transit system in a software to make it as a practical scenario.

For simulation of city bus transit system, we consider the peak hour and regular hour scenarios mentioned in Fig. 4. For simplicity, we focus to model the city bus transit system consisting of three bus stops covering 2 road segments. Total of 8 city buses were considered in a city bus system, from which, 4 buses follow one route and the other 4 buses travel along the other route. The varying velocities are considered according to the defined regular and peak hours of traffic as well as for the turning points. City buses move with speed of 16-18 meters/sec during peak hours of traffic and 20-22 meters/sec in regular hours of traffic. Similarly, the turning velocities of city buses during the peak hours of traffic are considered between 3-4 meters/sec and the turning speeds of buses during the regular hours is take in between 6-7 meters/sec. The headway characteristics of one minute between the city buses of route 1 as well as between the buses of route 2 were also considered while designing the system. The northbound direction of city buses is considered to generate the historic data comprising of time instants, bus IDs, route IDs and bus positions, for four days. The data is generated for total of 15 minutes per day.

\section{A. LSTM-based resource allocation algorithm}

To train the DL-based LSTM network for RB allocation in $\mathrm{mSc}$ network, generation of ground truth labels is required. 


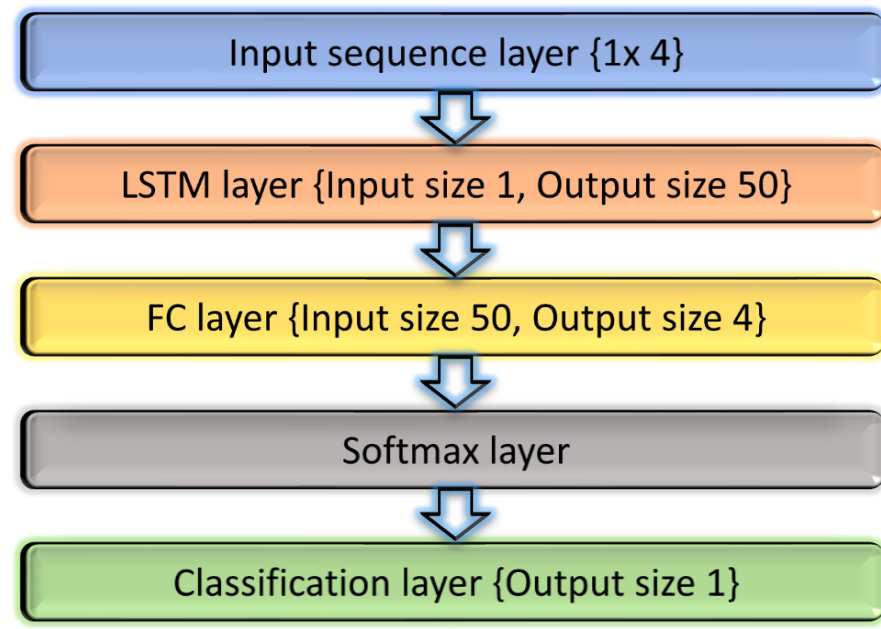

Fig. 5. LSTM architecture for resource allocation in mSc network.

Towards this goal, we consider the approach proposed in [16] for determination of interference patterns between $\mathrm{mScs}$ at each time instant to form the Time Interval Dependent Interference Graph (TIDIG). The historic data is used to find the interference relationship among mScs. Afterwards, the graph coloring mechanism was considered to allocate the RBs to mScs forming the TIDIG while considering the minimum demands of mobile users. The generation of ground truth labels is done for a whole day considering all the road segments associated to the city bus system. The channel conditions are considered as quasi static for 1 second. We assumed that each mobile user requires one $\mathrm{RB}$ and a single mobile user is connected to each of the mSc. As there exists total 8 city buses in a considered network and each city bus is equipped with one $\mathrm{mSc}$, so we have total of $8 \mathrm{mScs}$ in our system model. Simulations for allocation of RBs (generation of ground truth labels) is done for total of 15 minutes in a day. We considered maximum power of $19 \mathrm{~mW}$ associated to each $\mathrm{mSc}$ because there exists larger distance between $\mathrm{mScs}$ deployed in city buses and the value of noise is taken as 8.0906e-16 Hz. Once the RBs are allocated, we use these as ground truth labels in the proposed algorithm. Therefore, we have the input historic data and their corresponding labels that are required to train the LSTM network. The training process continues until the error between the LSTM predicted output and the optimized output is reduced. The error reduction is achieved by adjusting the weights of the network. The whole training process of a network is considered as an offline process. The LSTM network is trained using one hidden layer comprising of total of 50 neurons. As, the predicting accuracy of a network depends on the epoch size and min-batch size, we have used total of 80 epochs and mini-batch size of 16 . Gradient Descent is considered as the optimization algorithm and regularization is used as the loss function while training the LSTM. The architecture of LSTM used to predict the allocation of RBs is presented in Fig. 5. Then, the testing of the learned LSTM is done using the testing data and the maximum accuracy of $98.2 \%$ is achieved.

The proposed algorithm is considered for allocation of re- sources in $\mathrm{mSc}$ network because it outperforms other machine learning (ML) and DL-based algorithms. For comparison of proposed algorithm with ML and DL-based methods, we considered $k$-Nearest Neighbor (kNN) classification algorithm and the Artificial Neural Network (ANN). Table. II presents the maximum accuracy achieved using the mentioned algorithms. It can be noted that the LSTM-based RB allocation outperforms both the ANN and kNN algorithm. The kNN provides the lowest accuracy in allocation of resources.

Furthermore, we have determined the average data rate of a mSc network using the DL and ML-based resource allocation algorithms. Fig. 6 provides the relationship between the interference range of $\mathrm{mScs}$ and the data rate achieved using kNN-based RA in mSc network, ANN-based allocation of resources in $\mathrm{mSc}$ network using ANN, and LSTM-based resource allocation to $\mathrm{mScs}$. Overall, it is observed that the data rate decreases with the increase in interference range of mScs. This happens because RA using DL and ML-based algorithms mispredict some of the interference relationships present between the $\mathrm{mScs}$ depending on the nature of algorithm. It is notable that the average data rate achieved using LSTM-based RA is $8.43 \%$ and $17.79 \%$ more as compared to data rate achieved using ANN-based and kNN-based allocation of resources. Moreover, the data rate achieved using ANNbased RA is $8.63 \%$ bettwer in comparison with RA using kNN because we have also seen in Table. II that ANN-based RA provides $7.56 \%$ more accurate allocation of RBs to $\mathrm{mScs}$ as compared to RA using kNN.

Therefore, it is clear that resource allocation using LSTM provides better results in terms of prediction accuracy, and achievable data rate as compared to other machine learning and deep learning-based resource allocation mechanisms.

Afterwards, we have compared the results of proposed method with existing resource allocation methods. Fig. 7 provides the relationship between interference range of $\mathrm{mScs}$ and the number of RBs allocated in a mSc network with the help of bar graph. The bar graph shows an upsurge in RB utilization in $\mathrm{mSc}$ network using TIDIG-based RA mechanism, RA using TIPDIG, GPSDIG-based allocation of resources, as well as the proposed LSTM-based RA methodology. It is to be noted that TIDIG-based allocation of resources utilized the maximum number of RBs as it allocates the optimal number of RBs to mScs. It is to be noted that the TIDIG graphbased allocation of resources utilized the maximum number of RBs as it allocates the optimal number of RBs to mScs. Whereas, RBs used by the proposed mechanism is slightly less than TIDIG-based RB allocation but more than the ones used by the Threshold Percentage Dependent Interference Graph (TPDIG)-based allocation of RBs and the RBs allocated using Global Positioning Dependent Interference Graph(GPSDIG)-

TABLE II

Comparison of ML \& DL-Based RA methods.

\begin{tabular}{|l|l|}
\hline Algorithm & Accuracy \\
\hline kNN & $86.57 \%$ \\
\hline LSTM & $98.2 \%$ \\
\hline ANN & $94.13 \%$ \\
\hline
\end{tabular}


TABLE III

COMPUTATIONAL SPEED COMPARISON OF LSTM-BASED RA USING VARIOUS RA METHODS.

\begin{tabular}{c|c|c|c|c}
\hline \hline & $\begin{array}{c}\text { LSTM-based RA } \\
\text { (Proposed) }\end{array}$ & GPSDIG-based RA & TIPDIG-based RA & TIDIG-based RA \\
\hline $\begin{array}{c}\text { Average CPU time for RA } \\
\text { Speed }\end{array}$ & $\begin{array}{c}2.421184 \mathrm{sec} \\
1 \mathrm{time}\end{array}$ & $\begin{array}{c}1.022113 \mathrm{sec} \\
\approx 0.5 \mathrm{times}\end{array}$ & $\begin{array}{c}9.940741 \mathrm{sec} \\
\approx 5 \text { times }\end{array}$ & $\begin{array}{c}18.182717 \\
\approx 9 \text { times }\end{array}$ \\
\hline \hline
\end{tabular}

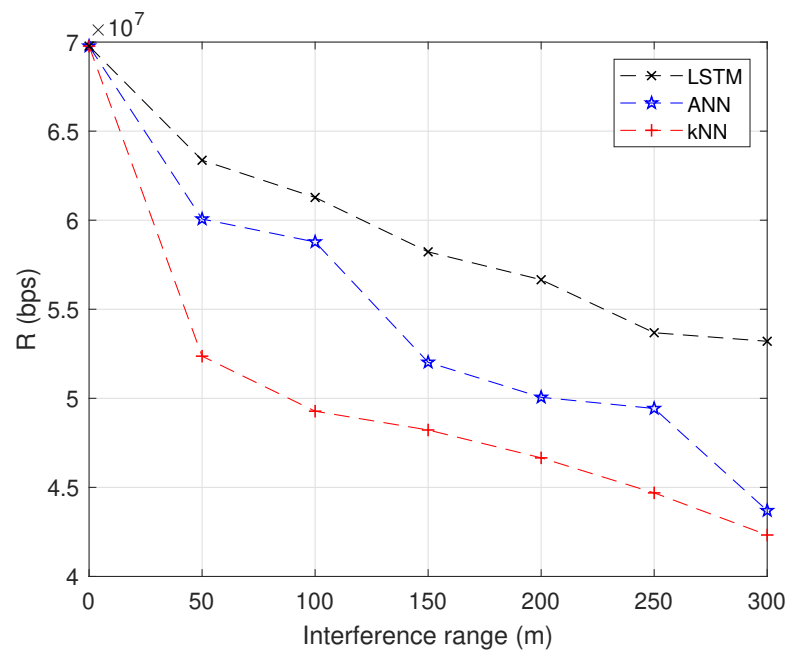

Fig. 6. Relationship between interference range and data rate of $\mathrm{mSc}$ network using DL and ML based RA.

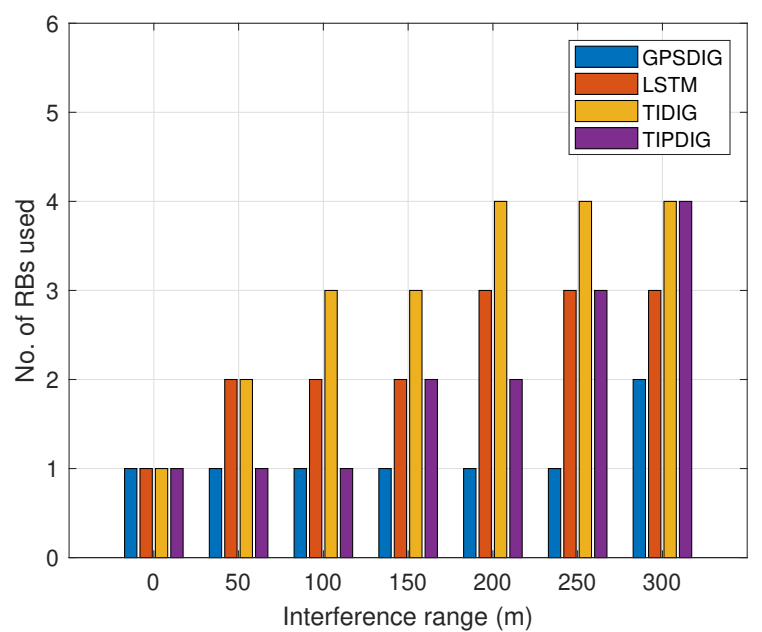

Fig. 7. Relationship between interference range and no. of RBs utilized in $\mathrm{mSc}$ network.

based. RBs utilized by proposed method is less than TIDIGbased

Fig. 8 represents the relation between interference range of $\mathrm{mSc}$ and data rate achieved using different $\mathrm{RB}$ allocation schemes. It is seen in the figure that data rate achieved using TIDIG-based RA stays almost the same over various values of interference range. This is due to the interference free nature of TIDIG where one RB is allocated to each individual $\mathrm{mSc}$ while diminishing the interference present between the $\mathrm{mScs}$. On the other hand, average data rate achieved using the proposed LSTM-based RA, GPSDIG-based allocation of RBs,

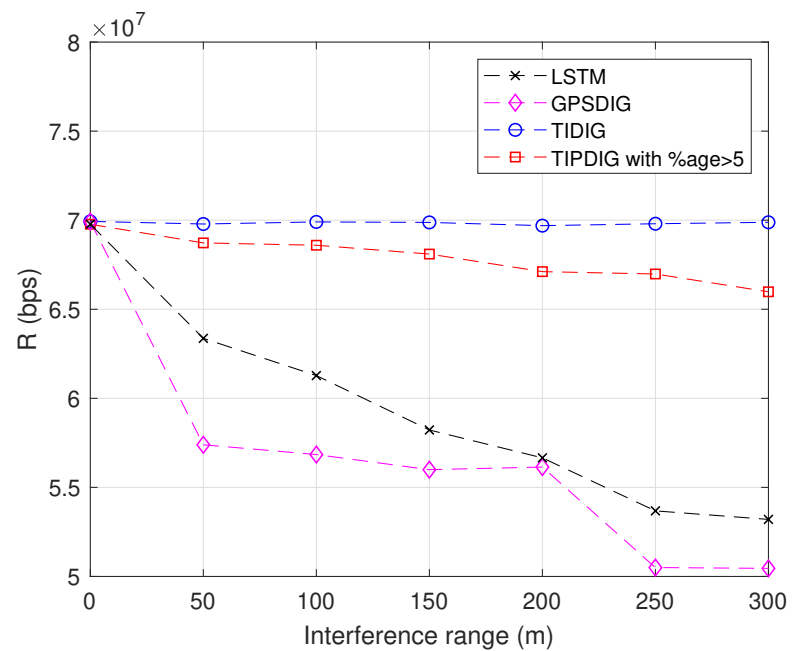

Fig. 8. Relationship between interference range and data rate of $\mathrm{mSc}$ network using proposed algorithm and various conventional approaches.

and RA using TIPDIG shows the deteriorating affect with the increase in interference range of $\mathrm{mScs}$. This is due to the nonconsideration of some of the interference relationships present between the mScs as GPSDIG-based allocation of RBs just considers the interference patterns between the $\mathrm{mScs}$ present at the start of time period and uses the same interference relationships for the whole time period. Similarly, in TIPDIGbased RA, percentage threshold is considered to diminish the effects of negligible interference patterns while allocating the RBs to mScs which simultaneously avoids interference to greater extent with escalation in interference range. Moreover, the proposed LSTM-based RA mechanism mispredicts some of the interference relationships present within the mScs. It is observed that the data rate achieved using TIDIG-based RA is $23.07 \%, 17.46 \%$, and $2.86 \%$ more as compared to GPSDIG, LSTM, and TIPDIG-based RA algorithms. Also, the data rate achieved using proposed LSTM-based allocation of resources is $12.43 \%$ less and $4.77 \%$ more in comparison with TIPDIGbased RA and RA using GPSDIG respectively.

The proposed methodology outperforms all other mentioned algorithms in terms of computational speed. Table. III provides the computational speed comparison of LSTMbased RB allocation method with various algorithms. In the proposed method, the RBs are allocated in much less time after training the network. The training process is done periodically according to the requirement. The RBs are allocated for a time period of whole day using the trained network. On the other hand, all other algorithms divides the time period in a number of time frames and allocation of resources is done at the beginning of each of the time frame. Each of them 
requires the generation of interference graph and various other computations while allocation of resources which makes them computationally expensive as well as they are expensive to implement too.

\section{CONCLUSION AND FUTURE WORK}

This paper examined the DL-based allocation of RBs to $\mathrm{mScs}$ integrated in city buses to serve the mobile users. LSTM neural networks are considered for RB allocation which demands for the historic data and their respective labels. The labels are generated using the optimization method that act as a supervisor in our algorithm. The network is trained by diminishing the error present between the predicted RBs allocated to $\mathrm{mScs}$ and the optimized RBs generated by the optimization algorithm. We have seen that LSTM provides better results than other machine and deep learning-based methods. It is also noticed that data rate achieved using the proposed algorithm is less in comparison with TIDIGbased RB allocation and TPDIG-based allocation of RBs. But, performs better than the GPSDIG-based results. It is also seen that LSTM-based algorithm is computationally less expensive than any other algorithm. It is also easy to implement as compared to other resource allocation mechanisms.

For future, data generation of realistic city bus transit system is under consideration in order to get the practical implementation of the proposed algorithm. Furthermore, unsupervised learning based model will be considered for allocation of RBs in $\mathrm{mSc}$ network.

\section{REFERENCES}

[1] "World Telecommunication Development Report 1999, Mobile Cellular," International Telecommunication Union, Tech. Rep., Oct. 1999. [Online]. Available: https://www.itu.int/ITU-D/ict/publications/wtdr_99/material/ wtdr99s.pdf.

[2] Ericsson. (2019) Mobility report. [Online]. Available: https://www.ericsson.com/4acd7e/assets/local/mobility-report/ documents/2019/emr-november-2019.pdf.

[3] Cisco. (2020) Cisco annual internet report. [Online]. Available: https://www.cisco.com/c/en/us/solutions/collateral/executiveperspectives/annual-internet-report/white-paper-c11-741490.html.

[4] A. Shahid, V. Maglogiannis, I. Ahmed, K. S. Kim, E. D. Poorter, and I. Moerman, "Energy-efficient resource allocation for ultra-dense licensed and unlicensed dual-access small cell networks," IEEE Trans. Mobile Comput., pp. 1-1, IEEE Early Access 2019.

[5] J. Rygaard, "Digitization, densification and why you should care," Nokia, Tech. Rep., Feb. 2017. [Online]. Available: https://www.nokia. $\mathrm{com} / \mathrm{blog} /$ digitization-densification-care/

[6] S. Jangsher and V. O. Li, "Resource allocation in moving small cell network," IEEE Trans. Wireless Commun., vol. 15, no. 7, pp. 45594570, 2016.

[7] S. Basnet, Y. He, E. Dutkiewicz, and B. A. Jayawickrama, "Resource allocation in moving and fixed general authorized access users in spectrum access system," IEEE Access, vol. 7, pp. 107863-107873, 2019.

[8] Z. Iftikhar, S. Jangsher, H. K. Qureshi, and M. Aloqaily, "Resource efficient allocation and RRH placement for backhaul of moving small cells," IEEE Access, vol. 7, pp. 47379-47 389, 2019.

[9] M. Kalil, A. Moubayed, A. Shami, and A. Al-Dweik, "Efficient lowcomplexity scheduler for wireless resource virtualization," IEEE Trans. Wireless Commun. Lett., vol. 5, no. 1, pp. 56-59, 2016.

[10] M. Kalil, A. Shami, A. Al-Dweik, and S. Muhaidat, "Low-complexity power-efficient schedulers for LTE uplink with delay-sensitive traffic," IEEE Trans. Veh. Technol., vol. 64, no. 10, pp. 4551-4564, 2015.

[11] M. Kalil, A. Al-Dweik, M. F. Abu Sharkh, A. Shami, and A. Refaey, "A framework for joint wireless network virtualization and cloud radio access networks for next generation wireless networks," IEEE Access, vol. 5, pp. 20814-20827, 2017.
[12] M. Kalil, A. Shami, and A. Al-Dweik, "QoS-aware power-efficient scheduler for LTE uplink," IEEE Trans. Mobile Comput., vol. 14, no. 8, pp. 1672-1685, 2015.

[13] H. Chen, D. Zhao, Q. Chen, and R. Chai, "Joint computation offloading and radio resource allocations in small-cell wireless cellular networks," IEEE Trans. Green Commun. Netw., pp. 1-1, 2020.

[14] C. Chen, R. A. Berry, M. L. Honig, and V. G. Subramanian, "The impact of unlicensed access on small-cell resource allocation," IEEE J. Sel. Areas Commun, pp. 1-1, IEEE Early Access 2020

[15] H. Zhang, K. Yang, and S. Zhang, "Resource allocation based on interference alignment with clustering for data stream maximization in dense small cell networks," IEEE Access, vol. 7, pp. 161 831-161 848, 2019.

[16] S. Zafar, S. Jangsher, M. Aloqaily, O. Bouachir, and J. B. Othman, "Resource allocation in moving small cell network using deep learning based interference determination," in 2019 IEEE 30th Annu. Int. Symp. on Personal, Indoor and Mobile Radio Communications (PIMRC). IEEE, 2019, pp. 1-6.

[17] S. Zafar, S. Jangsher, O. Bouachir, M. Aloqaily, and J. B. Othman, "QoS enhancement with deep learning-based interference prediction in mobile IoT," J. Comput. Commun., vol. 148, pp. 86-97, 2019.

[18] Z. Zhao, X. Cheng, M. Wen, B. Jiao, and C.-X. Wang, "Channel estimation schemes for IEEE $802.11 \mathrm{p}$ standard," IEEE Trans. Intell. Transp. Syst. Mag., vol. 5, no. 4, pp. 38-49, 2013.

[19] Z. Xiao, X. Shen, F. Zeng, V. Havyarimana, D. Wang, W. Chen, and $\mathrm{K}$. Li, "Spectrum resource sharing in heterogeneous vehicular networks: A noncooperative game-theoretic approach with correlated equilibrium," IEEE Trans. Veh. Technol., vol. 67, no. 10, pp. 9449-9458, 2018.

[20] S. Jaffry, S. F. Hasan, and X. Gui, "Efficient resource-sharing algorithms for mobile-cell's sidehaul and access links," IEEE Netw. Lett., vol. 1, no. 2 , pp. $72-75,2019$.

[21] F. Zhou, X. Zhang, R. Q. Hu, A. Papathanassiou, and W. Meng, "Resource allocation based on deep neural networks for cognitive radio networks," in 2018 IEEE/CIC Int. Conf. on Commun. in China (ICCC), Aug 2018, pp. 40-45.

[22] L. Liang, H. Ye, G. Yu, and G. Y. Li, "Deep-learning-based wireless resource allocation with application to vehicular networks," Proceedings of the IEEE, vol. 108, no. 2, pp. 341-356, Feb 2020.

[23] C. Qiu, H. Yao, F. R. Yu, F. Xu, and C. Zhao, "Deep Q-learning aided networking, caching, and computing resources allocation in softwaredefined satellite-terrestrial networks," IEEE Trans. Veh. Technol., vol. 68 , no. 6, pp. 5871-5883, June 2019.

[24] C. Wu, 1. Zhang, Q. Li, Z. Fu, W. Zhu, and Y. Zhang, "Enabling flexible resource allocation in mobile deep learning systems," IEEE Trans. Parallel Distrib. Syst., vol. 30, no. 2, pp. 346-360, Feb 2019.

[25] N. V. Huynh, D. T. Hoang, D. N. Nguyen, and E. Dutkiewicz, "Optimal and fast real-time resource slicing with deep dueling neural networks," IEEE J. Sel. Areas Commun, vol. 37, no. 6, pp. 1455-1470, June 2019.

[26] N. Zhao, Y. Liang, D. Niyato, Y. Pei, M. Wu, and Y. Jiang, "Deep reinforcement learning for user association and resource allocation in heterogeneous cellular networks," IEEE Trans. Wireless Commun., vol. 18, no. 11, pp. 5141-5152, Nov 2019.

[27] H. Lee, J. Kim, and J. Lee, "Resource allocation in wireless networks with deep reinforcement learning: A circumstance-independent approach," IEEE Syst. J., pp. 1-04, 2019.

[28] M. Eisen, C. Zhang, L. F. O. Chamon, D. D. Lee, and A. Ribeiro, "Learning optimal resource allocations in wireless systems," IEEE Trans. Signal Process., vol. 67, no. 10, pp. 2775-2790, May 2019.

[29] Y. Liu, H. Yu, S. Xie, and Y. Zhang, "Deep reinforcement learning for offloading and resource allocation in vehicle edge computing and networks," IEEE Trans. Veh. Technol., vol. 68, no. 11, pp. 11 158-11 168, Nov 2019.

[30] J. Wang, L. Zhao, J. Liu, and N. Kato, "Smart resource allocation for mobile edge computing: A deep reinforcement learning approach," IEEE Trans. Emerg. Topics Comput., pp. 1-1, 2019.

[31] X. He, K. Wang, H. Huang, T. Miyazaki, Y. Wang, and S. Guo, "Green resource allocation based on deep reinforcement learning in contentcentric IoT," IEEE Trans. Emerg. Topics Comput., pp. 1-1, 2018.

[32] J. Zhao, Y. Zhang, Y. Nie, and J. Liu, "Intelligent resource allocation for train-to-train communication: A multi-agent deep reinforcement learning approach," IEEE Access, vol. 8, pp. 8032-8040, 2020.

[33] S. Zafar, R. Hussain, F. Hussain, and S. Jangsher, "Interplay between Big Spectrum Data and mobile Internet of Things: Current solutions and future challenges," J. Comput. Netw., vol. 163, p. 106879, 2019.

[34] D. B. West et al., Introduction to graph theory. Prentice hall Upper Saddle River, NJ, 1996, vol. 2. 
[35] S. Xingjian, Z. Chen, H. Wang, D.-Y. Yeung, W.-K. Wong, and W.c. Woo, "Convolutional LSTM network: A machine learning approach for precipitation nowcasting," in Adv Neural Inf Process Syst, 2015, pp. 802-810.

[36] H. Yin and S. Alamouti, "OFDMA: A broadband wireless access technology," in 2006 IEEE sarnoff symp. IEEE, 2006, pp. 1-4.

[37] A. Iyer, C. Rosenberg, and A. Karnik, "What is the right model for wireless channel interference?" IEEE Trans. Wireless Commun., vol. 8, no. 5, pp. 2662-2671, 2009.

\section{BIOGRAPHIES}

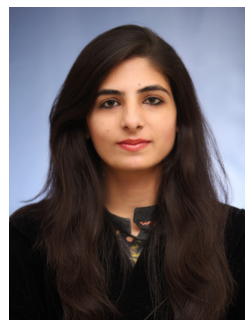

Saniya Zafar received her BS degree in Electrical (Telecommunication) Engineering from COMSATS Islamabad, Pakistan and MS (magna cum laude) degree in Electrical Engineering degree from Institute of Space Technology (IST) Islamabad, Pakistan in 2016 and 2018 respectively. She did her MS thesis on "Resource Allocation in Moving Small Cell Network using Deep Learning based location prediction" under the supervision of Dr. Sobia Jangsher. She is currently pursuing her $\mathrm{PhD}$ degree in Electrical Engineering from Institute of Space Technology Islamabad, Pakistan under the supervision of Dr. Sobia Jangsher She is interested in research areas including Big Data, Internet of Things (IoT), Moving Small Cells, Machine Learning, and Vehicular Networks.

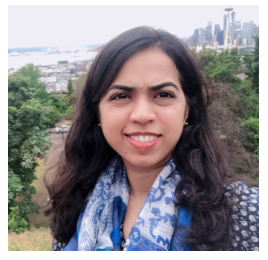

Sobia Jangsher received her B.E. degree in electronics engineering and M.S. in communication system engineering from National University of Science and Technology (NUST), Pakistan and $\mathrm{PhD}$ in Wireless Communication from The University of Hong Kong, Hong Kong. She did her M.S. thesis on "Adaptive transmission of video over MIMO channels" under the supervision of Dr. Syed Ali Khayam and $\mathrm{PhD}$ thesis on "Resource Allocation in Moving Small Cell Network" under the supervision of Prof. Victor O.K Li. She is currently working as an Assistant Professor in Institute of Space Technology, Islamabad, Pakistan. Her research mainly focuses on resource allocation in future wireless communication systems.

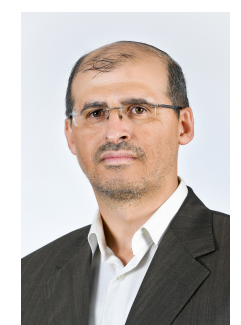

Arafat Al-Dweik (Senior Member, IEEE) received the B.Sc. degree in telecommunication engineering from Yarmouk University, Jordan, in 1994, and the M.S. (summa cum laude) and Ph.D. (magna cum laude) degrees in electrical engineering from Cleveland State University, Cleveland, OH, USA, in 1998 and 2001, respectively. He was with Efficient Channel Coding, Inc., Cleveland, from 1999 to 2001, where he was a Research and Development Engineer working on advanced modulation, coding, and synchronization techniques. From 2001 to 2003, he was the Head of the Department of Information Technology, Arab American University, Palestine. From 2003 to 2012, he was with the Communications Engineering Department, Khalifa University, UAE. From 2013 to 2014, he was an Associate Professor with the University of Guelph, Guelph, ON, Canada. He has been a Visiting Research Fellow with the School of Electrical, Electronic and Computer Engineering, Newcastle University, Newcastle upon Tyne, U.K., since 2006. He is also a Research Professor and a Member of the School of Graduate Studies, Western University, London, ON, Canada. He has received several research awards and he was a recipient of the Fulbright Scholarship from 1997 to 1999 . He was a TPC Member in several major conferences, such as IEEE GLOBECOM, ICC, PIMRC, and WCNC. He has extensive editorial experience where he serves as an Associate Editor for the IEEE TRANSACTIONS ON VEHICULAR TECHNOLOGY and IET Communications. 